\title{
Effect of geometrical symmetry on the angular dependence of the critical magnetic field in superconductor/normal metal multilayers
}

\author{
S. L. Prischepa ${ }^{1,2}$ C. Cirillo, ${ }^{2}$ V. N. Kushnir,${ }^{1}$ E. A. Ilyina, ${ }^{1}$ M. Salvato, ${ }^{2}$ and C. Attanasio ${ }^{2}$ \\ ${ }^{1}$ State University of Informatics and RadioElectronics, P. Brovka str. 6 Minsk 220013, Belarus \\ ${ }^{2}$ Dipartimento di Fisica "E.R. Caianiello" and Laboratorio Regionale SuperMat, INFM Salerno, \\ Università degli Studi di Salerno, Baronissi (Sa), I-84081, Italy
}

(Received 1 April 2005; published 22 July 2005)

\begin{abstract}
The angular dependence of the upper critical field, $H_{\mathrm{c}}(\Theta)$, for $\mathrm{Nb} / \mathrm{Pd}$ multilayers with different geometrical symmetries (i.e., with an even or an odd number of bilayers, $\left.N_{\text {bil }}\right)$ and thickness of $\mathrm{Nb}$ and Pd layers of the order of superconducting coherence length were investigated (the angle $\Theta$ is here defined from the plane parallel to the sample surface). The presence of an angular dimensional crossover at some temperature dependent angle $\Theta^{*}(T)$ was established. At angles $\Theta<\Theta^{*}(T)$ the multilayers are in two-dimensional mode while at $\Theta>\Theta^{*}(T)$ they are in a three-dimensional one. In addition, the sample with odd $N_{\text {bil }}$ has a larger value of $\Theta^{*}(T)$ with respect to the sample with even $N_{\text {bil }}$. The experimental results have been qualitatively explained in the framework of the Ginzburg-Landau theory. It was shown that the main features of the $H_{\mathrm{c}}(\Theta)$ dependences in samples with even and odd $N_{\text {bil }}$ values are related to the different localizations of the superconducting order parameter.
\end{abstract}

DOI: 10.1103/PhysRevB.72.024535

PACS number(s): 74.78.Fk, 74.20.De, 74.45.+c

\section{INTRODUCTION}

Considerable effort has been applied to study systems with strong anisotropy. The main reason for these investigations is the unique physical properties of anisotropic objects. Among them layered superconductors have attracted great attention due to the possibility to study the effect of anisotropy and the coupling nature between the superconducting layers on the magnetic phase diagram, vortex and pinning properties, superconductivity nucleation, etc. (see, e.g., Ref. 1 for reviews). From this point of view the periodic superconductor/normal metal $(\mathrm{S} / \mathrm{N})$ multilayers prepared by layer-by-layer vacuum deposition are still very interesting systems. The critical parameters of $\mathrm{S} / \mathrm{N}$ periodic structures differ from the corresponding quantities for thin films and anisotropic superconductors. Varying the layer thickness and the $\mathrm{S}$ and $\mathrm{N}$ materials it is possible to study both the proximity effect between the different metals in coupled multilayers and the behavior of the lattice of uncoupled superconducting films of different dimensionality.

One of the fundamental quantities characterizing the superconducting state is the upper critical magnetic field $H_{\mathrm{c}}$, and many efforts have been applied to study its temperature and angular dependences in different $\mathrm{S} / \mathrm{N}$ systems. ${ }^{2-12}$ In particular, rather comprehensive understanding of the nature of the dimensional crossover-namely, the transition from three-dimensional (3D) to two-dimensional (2D) mode upon a variation of the temperature $T$-leads to deeper insight into the interaction between the superconducting layers and into the superconducting phase nucleation. ${ }^{13-15}$ Recently, ${ }^{16,17}$ we have shown that the dimensionality of finite $\mathrm{S} / \mathrm{N}$ multilayers can be influenced by the "odd-even" effect related to the sample finiteness (in particular, the number of bilayers, odd or even) which determines the nucleation position of the superconducting phase. ${ }^{18}$ The angular dependence of $H_{\mathrm{c}}$ can be of highest importance to get additional useful information about the structure of multilayers, about the interaction between $\mathrm{S}$ layers as well as between the adjacent $\mathrm{S}$ and $\mathrm{N}$ layers, and about the origin of the superconducting phase nucleation in anisotropic superconductors. ${ }^{19}$

Originally interest in the $H_{\mathrm{c}}(\Theta)$ study $(\Theta$ being the angle between the applied magnetic field and the plane of the sample) was pushed by the possibility to be an alternative test of the dimensionality of superconductivity and an additional opportunity to study the influence of the interface boundaries on the properties of the superconducting phase. The fundamental results concerning the $H_{\mathrm{c}}(\Theta)$ dependences in homogeneous superconductors were obtained on the basis of the Ginzburg-Landau (GL) theory. ${ }^{20-23}$ For infinite anisotropic superconductors the dependence $H_{\mathrm{c}}(\Theta)$ is a smooth curve in the whole $\Theta$ range (including $\Theta=0$ ). This curve is easily reproduced within the anisotropic effective mass GL theory: ${ }^{23}$

$$
H_{c}^{3 D}(\Theta)=H_{c}(0) /\left(\cos ^{2} \Theta+\gamma^{2} \sin ^{2} \Theta\right)^{1 / 2},
$$

where $\gamma=H_{c}(0) / H_{c}(\pi / 2)$ is the anisotropy parameter. For superconductors with two flat surfaces Tinkham derived the formula for the $H_{\mathrm{c}}(\Theta)$ dependence in the thin-film 2D limit $(d \ll \xi),{ }^{21}$ which gives a discontinuous derivative $\partial H_{\mathrm{c}}(\Theta) / \partial \Theta$ at $\Theta=0$,

$$
\left|\frac{H_{c}^{2 D}(\Theta) \sin \Theta}{H_{c}(\pi / 2)}\right|+\left[\frac{H_{c}^{2 D}(\Theta) \cos \Theta}{H_{c}(0)}\right]^{2}=1 .
$$

It was established also that the $H_{\mathrm{c}}(\Theta)$ shape and nucleation position of the superconducting phase are mutually related to each other. In the thin-film limit the nucleation always starts at the center of the slab. ${ }^{22}$ At large sample thickness the nucleation starts apart from the center and reaches the familiar surface superconductivity situation. ${ }^{20,24}$

The layered structure of superconducting and nonsuperconducting films of finite thickness creates additional diffi- 
culties for understanding of the angular dependences of the upper critical magnetic field. For example, in $\mathrm{S} / \mathrm{N}$ multilayers in $2 \mathrm{D}$ mode [according to the temperature dependence of the parallel critical magnetic field, $\left.H_{\mathrm{c}}(T, \Theta=0)\right]$ the $H_{\mathrm{c}}(\Theta)$ points fall faster with angle growth with respect to Tinkham's expression (2) for thin films. ${ }^{2,3}$ Moreover, in the temperature region of linear $H_{\mathrm{c}}(T, \Theta=0)$ dependence (where the $3 \mathrm{D}$ mode is realized), the angular dependences reveal the pronounced cusp, ${ }^{2,25,26}$ which is a feature of the $2 \mathrm{D}$ regime. To resolve these discrepancies between theoretical descriptions of homogeneous superconductors and experiments on multilayers, Glazman proposed to take into account both the discrete structure of multilayers and the finite coupling interaction between individual $\mathrm{S}$ layers in the sample. ${ }^{27}$ As a result, for certain values of superconducting layer thickness and of the thickness of interlayers separating them, the dimensional crossover could be observed on the angular dependence $H_{c}(\Theta)$ when the $3 \mathrm{D}$ mode at $\Theta=\pi / 2$ changes smoothly into $2 \mathrm{D}$ dependence as the angle $\Theta$ decreases to zero. $^{12,28-30}$

Apart from this aspect other theoretical attempts have been done to describe the whole $H_{\mathrm{c}}(\Theta)$ curve mainly for infinite multilayers of different coupling nature between $\mathrm{S}$ layers. ${ }^{31-36}$ On the other hand, the finiteness of the multilayers samples (i.e., the precise position of the superconducting nucleus) has been found to be a very important parameter in determining, for example, the resistive transition to the superconducting state ${ }^{16}$ and the shape of the $H-T$ phase diagram. ${ }^{17}$ From this viewpoint the survey of the $H_{\mathrm{c}}(\Theta)$ characteristics in multilayers with the possibility of finetuning the position of superconducting nucleus is of great significance at least for two reasons: first, because of the mutual relation between the $H_{\mathrm{c}}(\Theta)$ shape and the nucleation position of the superconducting phase and, second, because in multilayers it is possible to precisely control, change, and know the position where the superconducting phase nucleates.

In this work we present the results of measurements of the $H_{\mathrm{c}}(\Theta)$ dependences for $\mathrm{Nb} / \mathrm{Pd}$ multilayers at different temperatures and we analyze the difference with the existing theoretical descriptions in order to deepen the understanding of the nature of the proximity coupling and of the wave function distribution in $\mathrm{S} / \mathrm{N}$ multilayered nanostructures. The $\mathrm{Nb} / \mathrm{Pd}$ system was chosen because of its pronounced interface transparency. ${ }^{37}$ We prepared $\mathrm{Nb} / \mathrm{Pd}$ samples with different geometrical symmetries in order to vary the position of the nucleation of the superconducting phase. ${ }^{17}$ The angular measurements were performed on the same set of samples analyzed in our previous work in which we reported only the results of the $H_{\mathrm{c}}$ temperature dependence. ${ }^{17}$ We performed angular measurements in the temperature range where, according to the $H_{\mathrm{c}}(T, \Theta=0)$ result, samples are in $2 \mathrm{D}$ mode. However, in agreement with previous experimental works, ${ }^{4,25,28}$ the measured $H_{\mathrm{c}}(\Theta)$ data were not described by the 2D Tinkham expression [Eq. (2)]. We show that, at fixed temperature, for the sample with the symmetry plane in the central $\mathrm{S}$ layer the $2 \mathrm{D}$ mode remains up to higher $\Theta$ values with respect to the characteristic of the sample in which the symmetry plane falls in the central $\mathrm{N}$ layer. We relate this fact to the more pronounced bidimensionality in the former case as was discussed in Refs. 16 and 17. We discuss the experimental method for the determination of the angle $\Theta^{*}$, the angle where the dimensional crossover occurs, and we estimate the $H_{\mathrm{c}}(\Theta)$ dependence for finite $\mathrm{S} / \mathrm{N}$ multilayers in the simplest way within the GL approach.

\section{EXPERIMENT}

The $\mathrm{Nb} / \mathrm{Pd}$ samples were grown on $\mathrm{Si}(100)$ substrates at room temperature by using a dual-source magnetically enhanced dc triode sputtering system with a movable substrate holder. ${ }^{11}$ The high-quality layered structure of the samples, with negligibly small interfacial roughness (order of $1 \mathrm{~nm}$ ), was confirmed by high- and low-angle x-ray diffraction measurements. ${ }^{11}$ A specially designed movable shutter allowed the simultaneous deposition of the two samples with a different number of bilayers, $N_{\text {bil }}{ }^{17}$ We have fabricated $\mathrm{Nb} / \mathrm{Pd}$ samples in the same deposition run with $N_{\text {bil }}=9$ and 10 , respectively. The relation between the thickness of $\mathrm{Pd}$ $\left(d_{\mathrm{N}}\right)$ and $\mathrm{Nb}\left(d_{\mathrm{S}}\right)$ layers was $d_{\mathrm{N}}=d_{\mathrm{S}} / 2=10 \mathrm{~nm}$. For both samples the top and bottom layers were made of the normal layer. This means that the sample with $N_{\text {bil }}=9(=10)$ consists of 9 (10) $\mathrm{Pd} / \mathrm{Nb}$ bilayers plus the capping $\mathrm{Pd}$ layer. As a result, the geometrical plane of the sample symmetry falls into the center of the central $\mathrm{S}$ layer for $N_{\text {bil }}=9$ (sample SP) and into the center of the central $\mathrm{N}$ layer for $N_{\mathrm{bil}}=10$ (sample NP). ${ }^{17}$ The fabrication of two samples in the same deposition run allows us to assume the same $\mathrm{Nb}$ and $\mathrm{Pd}$ properties in both samples as well as the same interface transparency, giving us the possibility to suppose geometrical symmetry as the only difference between the two systems.

We performed dc transport measurements with a standard four-probe technique with a variable orientation of the external magnetic field which was created by a superconducting solenoid. The accuracy of the rotation angle was $\pm 0.1^{\circ}$. The $H_{\mathrm{c}}$ values were extracted from the $R(H)$ curves measured at the onset of the superconducting transition. The temperature stabilization during the measurements was $\pm 0.01 \mathrm{~K}$. More details about the properties of these samples were reported elsewhere. ${ }^{11,17}$

\section{RESULTS}

In Ref. 17 we published the results of a detailed study of the $H-T$ phase diagrams for these samples. We established that the sample NP reveals the classical $H_{\mathrm{c}}(T, \Theta=0)$ dependence for $\mathrm{S} / \mathrm{N}$ multilayers having $d_{\mathrm{S}} \sim d_{\mathrm{N}} \sim \xi-$ i.e., the presence of temperature dimensional crossover at temperature $T^{*}=3.60 \mathrm{~K}$ [Fig. 2(a) of Ref. 17]. At $T<T^{*}, H_{\mathrm{c}}(T, \Theta=0)$ $\sim\left(T_{\mathrm{c}}{ }^{2 \mathrm{D}}-T\right)^{1 / 2}\left(T_{\mathrm{c}}^{2 \mathrm{D}}\right.$ being the critical temperature in the $2 \mathrm{D}$ mode), which is a signature of bidimensionality, while at $T$ $>T^{*}$ multilayer behaves like a $3 \mathrm{D}$ system and $H_{\mathrm{c}}(T, \Theta=0)$ $\sim\left(T_{\mathrm{c}}-T\right) .{ }^{1}$ On the other hand, sample SP did not reveal the pronounced linear part in the $H_{\mathrm{c}}(T, \Theta=0)$ dependence [Fig. 2(b) of Ref. 17] and the $H_{\mathrm{c}}(T, \Theta=0)$ curve was square root like in the whole temperature range, even close to $T_{\mathrm{c}}$. Such a difference is related to the different symmetries of the order parameter describing the two systems. At $T$ close to $T_{\mathrm{c}}$ in the 
sample SP the superconducting nucleus appears in the central $S$ layer (the nucleation position is $z_{0}=0$ ) and this situation remains unchanged in the entire temperature range $0<T$ $<T_{\mathrm{c}}$. For the sample NP for $T^{*}<T<T_{\mathrm{c}}$ the superconducting nucleus is spread between two central $\mathrm{S}$ layers while for $T$ $<T^{*}$ it is shifted to one of the $\mathrm{S}$ layers adjacent to the central $\mathrm{N}$ layer, changing the position of the nucleation from $z_{0}=0$ (at $T^{*}<T<T_{\mathrm{c}}$ ) to $z_{0}= \pm \Lambda / 2\left(\right.$ at $\left.T<T^{*}\right)$. Here $\Lambda=d_{\mathrm{S}}+d_{\mathrm{N}}$ is the period of the layered structure. This change of symmetry of the order parameter in the sample NP causes a sharp 2D-3D crossover. ${ }^{17}$

In this paper we first show the results for $H_{\mathrm{c}}(\Theta)$ for the sample NP. We performed angular measurements in the temperature range $1.8 \mathrm{~K}<T<T^{*}$. In Fig. 1 such characteristics measured at three fixed temperatures $T=3.45 \mathrm{~K}, 2.91 \mathrm{~K}$ and $2.05 \mathrm{~K}$ are presented. The general features of all curves is the presence of a cusp at $\Theta=0$. This behavior has been obtained at all the other measured temperatures. At first glance, this result is a consequence of the bidimensional behavior of $H_{\mathrm{c}}(T, \Theta=0)$ at $T<T^{*}$, but Tinkham's expression (2) does not fit the experimental data (dashed curves in Fig. 1). As was shown for S/I superlattices, ${ }^{27,28}$ the reason is the incorrect application of Eq. (2) for describing the whole $H_{\mathrm{c}}(\Theta)$ dependence. In fact, the $H_{\mathrm{c}}(\Theta)$ curve is divided into two parts which are related to two different dimensionalities of the sample even at $T<T^{*}$. In other words, an angle $\Theta^{*}$ exists such that, when $\Theta<\Theta^{*}$, the experimental dependence is well described by Tinkham's expression in which, however, the $H_{\mathrm{c}}(\pi / 2)$ value is a free parameter and it is smaller than the measured value of the perpendicular critical magnetic field. In our case this fit is shown for each temperature as a solid line in Fig. 1. For $\Theta>\Theta^{*}$, in the case of S/I superlattices, the 3D mode takes place and the angular dependence of $H_{\mathrm{c}}$ is described by Eq. (1). ${ }^{28}$ The 3D-2D angular crossover happens in this case smoothly. In our sample, as is seen from Fig. 1, the situation is different. At $T=3.45 \mathrm{~K}$ a sharp transition takes place at the angle $\Theta^{*}$. Actually the same is valid also for $T=2.91 \mathrm{~K}$ and $T=2.05 \mathrm{~K}$. Another peculiarity of this sample is related to the unusual 3D mode at $\Theta>\Theta^{*}$. For the sake of convenience we show the Lawrence-Doniach fit [Eq. (1)] only for one temperature [short-dotted line in Fig. 1(a)]. As can be seen this curve corresponds well to the experimental data only in the tail of the curve when $\Theta$ approaches $\pi / 2$. At the same time when using Eq. (2) with $H_{\mathrm{c}}(0)$ as a free parameter we obtain very good correspondence to the experimental data [dash-dotted line in Fig. 1(a)]. The physical reason for this result will be discussed later.

In Fig. 2 we show the $H_{\mathrm{c}}(\Theta)$ curves measured at three temperatures $T=3.50,2.50$, and $1.91 \mathrm{~K}$ for sample SP. By the dashed lines we show the result of Tinkham's expression (2), by the solid line the result of the Tinkham fit, but with $H_{\mathrm{c}}(\pi / 2)$ as a free parameter, and by the dash-dotted line the result of the Tinkham fit with $H_{\mathrm{c}}(0)$ as a free parameter. As follows from Fig. 2, the sample SP also presents the angular crossover, but the sharp change at $\Theta^{*}$ does not arise. Another peculiarity of this sample is the noticeably larger $\Theta^{*}$ values and the smaller deviation of the experimental data from the Tinkham curve (2) obtained without fitting parameters. Both these facts indicate that sample SP, with the symmetry plane
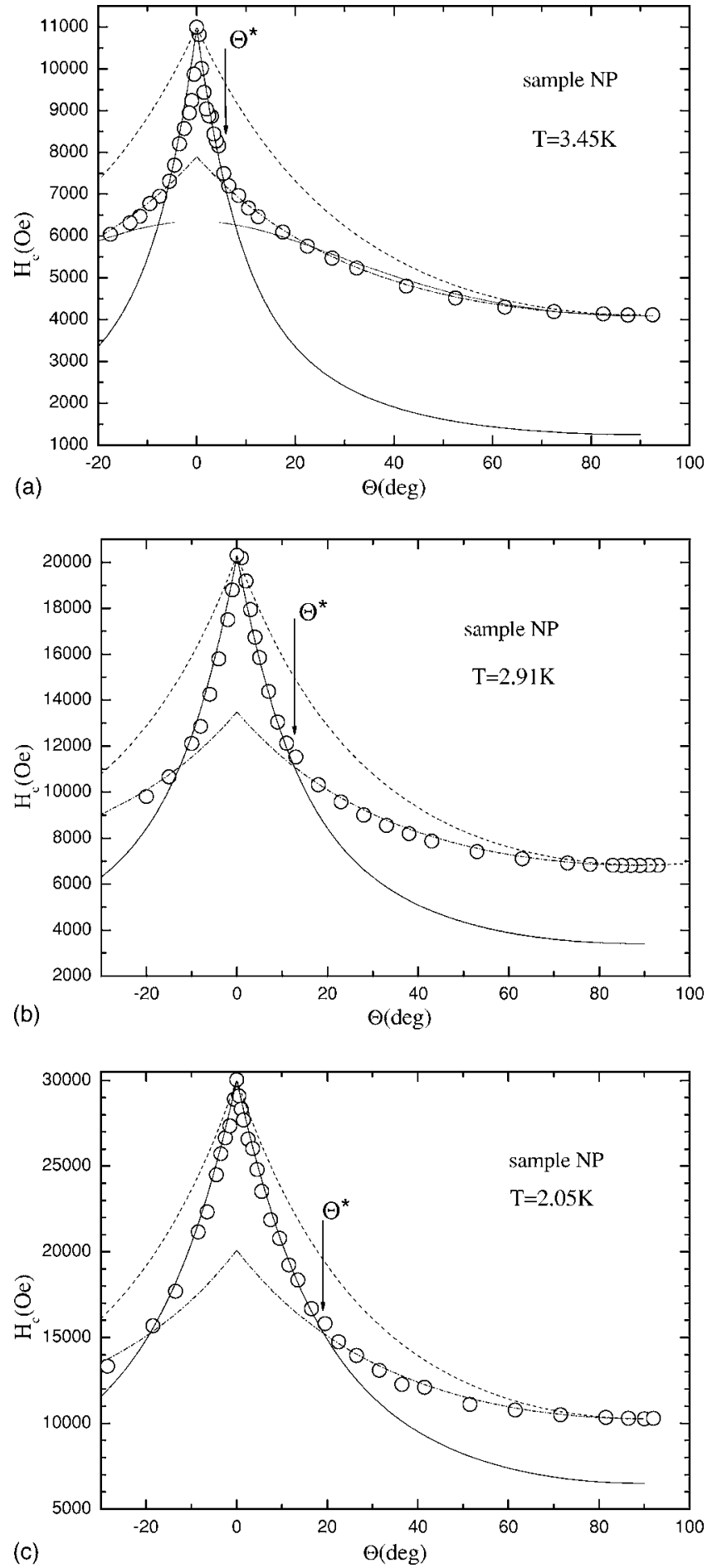

FIG. 1. Angular dependences of the upper critical magnetic field for sample NP. See text for the meaning of the different lines. Arrows indicate the value of $\Theta^{*}$ for each temperature: (a) $T=3.45 \mathrm{~K}$, (b) $T=2.91 \mathrm{~K}$, and (c) $T=2.05 \mathrm{~K}$.

in the center of the middle $\mathrm{S}$ layer, is "more" bidimensional than sample NP also from the viewpoint of the $H_{\mathrm{c}}(\Theta)$ dependence, in agreement with the $H_{\mathrm{c}}(T, \Theta=0)$ result. ${ }^{17}$

In Fig. 3 we plot the relative deviation of the experimental data from Eq. (2), $\delta=\left[H_{\mathrm{c}}(\Theta) / H_{\mathrm{c}}^{2 \mathrm{D}}(\Theta)\right]^{2}$, as a function of $\Theta$ (Ref. 38) for sample NP. When the magnetic field is directed 

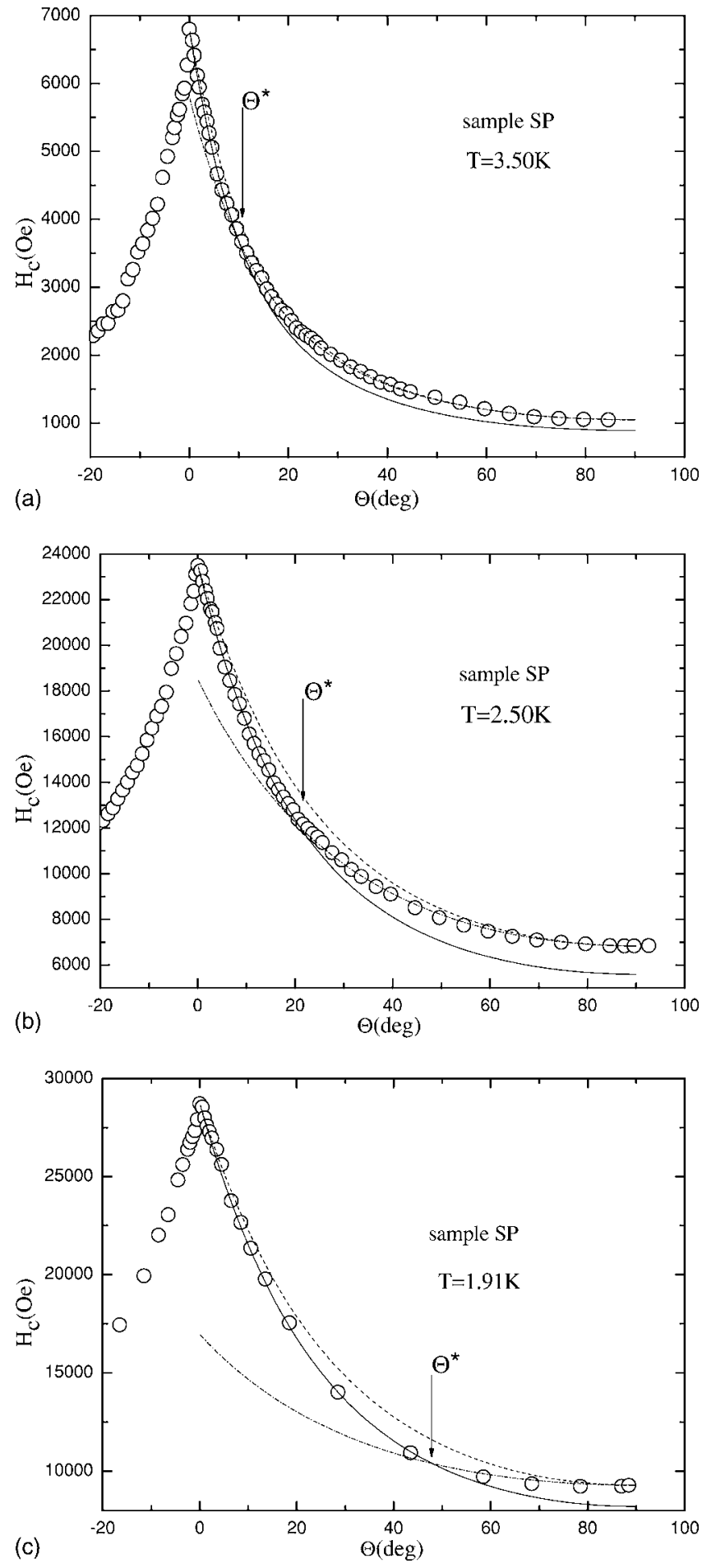

FIG. 2. Angular dependences of the upper critical magnetic field for sample SP. See text for the meaning of the different lines. Arrows indicate the value of $\Theta^{*}$ for each temperature: (a) $T=3.50 \mathrm{~K}$, (b) $T=2.50 \mathrm{~K}$, and (c) $T=1.91 \mathrm{~K}$.

along the basal plane of the substrate or along the perpendicular direction, $\delta=1$, since the experimental values of $H_{\mathrm{c}}(0)$ and $H_{\mathrm{c}}(\pi / 2)$ were used as parameters for Eq. (2). The shape of the deviation as a function of the angle is similar for all temperatures, but the angle $\Theta_{\max }$, at which the deviation

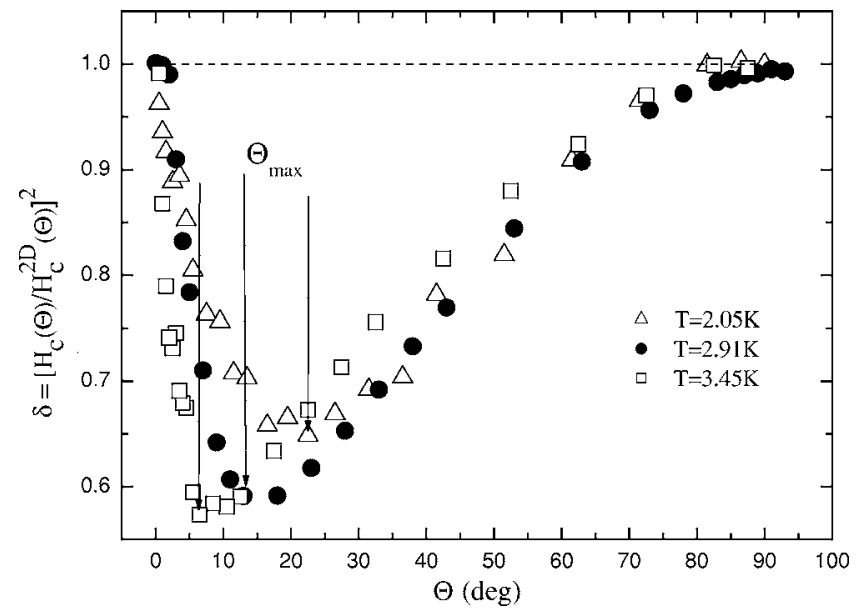

FIG. 3. Relative deviation $\delta=\left[H_{\mathrm{c}}(\Theta) / H_{\mathrm{c}}^{2 \mathrm{D}}(\Theta)\right]^{2}$ as a function of $\Theta$ for sample NP at three different temperatures. Arrows indicate the values of $\Theta_{\max }$ for each temperature.

$\Delta \delta(\Theta)=1-\delta(\Theta)$ reaches its maximum value, is temperature dependent.

In Fig. 4 by open (solid) symbols we show the $\Theta^{*}$ and $\Theta_{\text {max }}$ temperature dependences for the sample SP (NP). As is seen, the values of $\Theta_{\max }$ well correspond to the values of $\Theta^{*}$. This result indicates that the analysis of the $\delta$ versus $\Theta$ plot could be a simple method for finding out $\Theta^{*} \equiv \Theta_{\max }$.

\section{DISCUSSION}

The obtained 2D mode at $\Theta<\Theta^{*}$ for sample NP naturally fits the physical picture proposed in Refs. 27 and 28 and is related to the coherence length values in the temperature range $2.0 \mathrm{~K}, \ldots, 3.6 \mathrm{~K}$. Indeed, from the $H_{\mathrm{c}}(T, \Theta=\pi / 2)$ dependence we may obtain the GL coherence length at zero temperature $\xi(0),{ }^{39}$ which in our case is equal to $12.6 \mathrm{~nm}$. From this we get that $\xi(T)=\xi(0)\left(1-T / T_{\mathrm{c}}\right)^{-1 / 2}$ changes from $\approx 18 \mathrm{~nm}$ at $T=2 \mathrm{~K}$ up to $\approx 33 \mathrm{~nm}$ at $T=3.60 \mathrm{~K}$-i.e., $\xi$ $\sim d_{\mathrm{S}}$ and $\xi \leqslant \Lambda$. This means that for $T<T^{*}$ the wave function

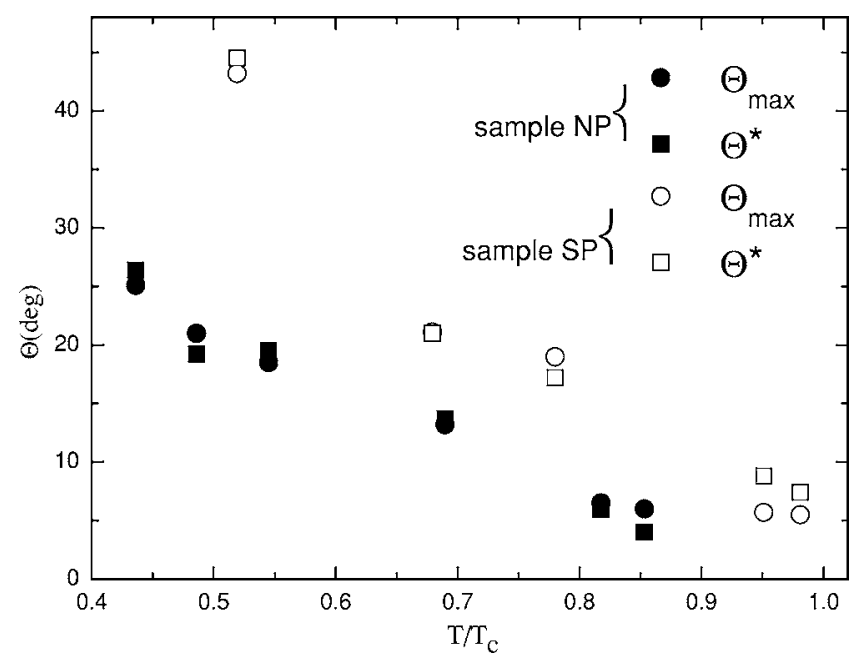

FIG. 4. Temperature dependences of $\Theta^{*}$ and $\Theta_{\max }$ for sample NP (solid symbols) and for sample SP (open symbols). 
of the superconducting condensate weakly changes with the thickness of the $\mathrm{S}$ layer, which is a signature of the 2D nature of superconductivity. When the sample is rotated from $\Theta$ $=0$ towards $\Theta^{*}$ the superconducting nucleus is still localized in one period of the $\mathrm{S} / \mathrm{N}$ structure and the $\mathrm{S} / \mathrm{N}$ interfaces favor the localization of the superconducting nucleus. For $\Theta>\Theta^{*}$ two factors influence the $H_{c}(\Theta)$ curves. First, the perpendicular component of the external magnetic field spreads the localization degree of the order parameter on more than one period. In this case the sample could not be concerned as 2D anymore. Second, the finite thickness of the sample determines the symmetry plane which falls in the middle of the central $\mathrm{N}$ layer. Consequently, in a perpendicular field the order parameter is symmetric. ${ }^{17}$ Due to this and due to $\xi$ values, the superconducting nucleus in the perpendicular field can be represented as dumbbell shaped, with two maxima in the adjacent central $\mathrm{S}$ layers. ${ }^{40}$ This is the characteristic feature of 3D objects as also shown by linear $H_{\mathrm{c}}(0)$ versus $T$ dependence ${ }^{17}$; but Eq. (1) is not valid in this case because it was deduced in the approximation of the homogeneous infinite medium. For our multilayers the superconducting order parameter, changing significantly in the whole sample, remains relatively homogeneous in one $\mathrm{S}$ layer. This probably explains the applicability of the Tinkham formula (2) also at $\Theta>\Theta^{*}$. So we get, from one side, at $\Theta>\Theta^{*}$ an inhomogeneous structure with bound $S$ layers (i.e., 3D structure), and from the other, at $\Theta<\Theta^{*}$, we get an analogous of the homogeneous thin film. $\Theta^{*}$ is the angle where this change of topology of the superconducting nucleus takes place.

Within the above-described qualitative picture one can estimate the angular dependences of the upper critical field for finite $\mathrm{S} / \mathrm{N}$ metal multilayers taking account of the nucleation position of the superconducting phase. Here we interpret the experimental results by estimating $H_{\mathrm{c}}(\Theta)$ within the simplest variation of the GL model, ${ }^{20-22,41}$ taking account of the real symmetry of the multilayers.

We chose the axis coordinates in the following way: the axis $O Z$ is directed perpendicular to the layer surface and the coordinate plane $X O Y$ is parallel to layers and coincides with the symmetry plane of the multilayer. The superconductor structure is supposed to be infinite along the $O X$ and $O Y$ directions and has thickness $L$ along the $O Z$ direction. The vector potential $\boldsymbol{A}(\boldsymbol{r}) \equiv(H z \cos \Theta-H y \sin \Theta, 0,0)$ is related to the external magnetic field $\boldsymbol{H} \equiv(0, H \cos \Theta, H \sin \Theta)$. Taking into account that in the critical region the GL wave function could be expressed as $\Psi(\mathbf{r})=e^{i k x} \psi(y, z)$, we may write the GL functional in the following way:

$$
\begin{aligned}
F= & \iint d y d z\left\{\left(\partial_{y} \psi\right)^{2}+\left(\partial_{z} \psi\right)^{2}-a(z, T) \psi^{2}\right. \\
& \left.+H^{2}\left(z \cos \Theta-z_{0}-y \sin \Theta\right)^{2} \psi^{2}\right\},
\end{aligned}
$$

where $z_{0} \equiv k / H_{0} ; a(z ; T)$ is the step function. ${ }^{18}$ We minimize Eq. (3), by choosing the probe wave function with separated variables: ${ }^{39}$

$$
\psi(y, z) \rightarrow \psi(y, z)=f(y) g(z),
$$

with the boundary conditions $f( \pm \infty)=0$ and

$$
g^{\prime}( \pm L / 2)=0 .
$$

Moreover, on $\mathrm{S} / \mathrm{N}$ interfaces the join condition has to be fulfilled, ${ }^{1,42}$

$$
\left.\frac{g^{\prime}}{g}\right|_{z_{i}+0}=\left.p_{i} \frac{g^{\prime}}{g}\right|_{z_{i}-0},
$$

where the quantities $p_{\mathrm{i}}$ determine the jump of the logarithmic derivative of the wave function. The expression for $g(z)$ would be

$$
g^{\prime \prime}(z)+\left[a(z, T)-H^{2} \cos ^{2} \Theta(z-\bar{z})^{2}-H|\sin \Theta|\right] g(z)=0,
$$

where $\bar{z}$ means the average over the distance. By solving Eqs. (5)-(7) one can find the required $H_{\mathrm{c}}(\Theta)$ dependence.

Moreover, from Eqs. (3) and (4) it is possible to extract the expression for the important quantity $\alpha(\Theta)$ $\equiv H_{c}^{-1}(\Theta) d H_{c}(\Theta) / d \Theta$, whose analytical properties at $\Theta=0$ determine the effective dimension of the superconducting nucleus. In fact, for $\Theta=0$ we get

$$
\alpha(\Theta= \pm 0)=-\operatorname{sgn}(\Theta) /\left[H_{c}(0) \sigma^{2}\right],
$$

where $\left.\sigma^{2} \equiv \overline{(z-\bar{z})^{2}}\right|_{\Theta=0}$.

From Eq. (8) it follows that within the approach used the relative drop of the $H_{\mathrm{c}}(\Theta)$ characteristic at $\Theta=0$ is determined only by the parameter of localization of the wave function $\sigma$ in the region of $\bar{z}$.

In order to check qualitatively the physical meaning of the model we performed the calculation of $H_{\mathrm{c}}(\Theta)$ for S/N multilayers with even $N_{\text {bil }}$. We chose $N_{\text {bil }}=4$ (plus a capping $\mathrm{N}$ layer) as a model object. We also performed the simulations for NSN trilayer (i.e., one $\mathrm{S} / \mathrm{N}$ bilayer) which is considered analogous to thin films. We assume $d_{\mathrm{N}}=0.5 d_{\mathrm{S}}=\xi_{\mathrm{S}}(0)$ $=2 \xi_{\mathrm{N}}\left(T_{\mathrm{c}}\right)$. Here $\xi_{\mathrm{S}(\mathrm{N})}$ is the value of the coherence length in the $\mathrm{S}(\mathrm{N})$ layer. The temperature dependences of the coherence lengths were assumed as $\xi_{\mathrm{S}}(T)=\xi_{\mathrm{S}}(0)\left(1-T / T_{\mathrm{S}}\right)^{-1 / 2}$ (Ref. 39) and $\xi_{\mathrm{N}}(T)=\xi_{\mathrm{N}}\left(T_{\mathrm{c}}\right)\left(T / T_{\mathrm{c}}\right)^{-1 / 2}$ (Ref. 43) (T $T_{\mathrm{s}}$ is the critical temperature of the bulk superconducting material). In Fig. 5 we show the calculated $H_{\mathrm{c}}(\Theta)$ characteristics at $T$ close to $T^{*}$. The result for the one bilayer sample exactly reproduces Tinkham's curve for a $2 \mathrm{D}$ homogeneous superconductor. In this case there is only one parameter $\bar{z}=0$ that corresponds to the upper critical field in the whole temperature range. The function $g(z)$ is symmetrical. At $\Theta=0$ the superconducting nucleus is situated in the symmetry plane of the sample. For the four bilayer structure the 2D behavior with function $g(z)$ localized in one $S$ layer realizes for $\Theta$ $<\Theta^{*}$ (open circles in Fig. 5); at $\Theta>\Theta^{*}$ the function $g(z)$ spreads over two $\mathrm{S}$ layers and the $H_{\mathrm{c}}(\Theta)$ behavior changes to 3D one (solid circles in Fig. 5).

The obtained results of numerical simulations illustrates the angular dimensional crossover and the influence of the nucleation position (parameter $\bar{z}$ ) and of the value of localization parameter $\sigma$ on the $H_{\mathrm{c}}(\Theta)$ shape. Indeed, the $2 \mathrm{D}$ behavior corresponds to $\sigma \leqslant d_{\mathrm{S}}$ and $\bar{z}$ falls into the center of one of S layers. ${ }^{16}$ From the experimental data of Figs. 1 and 2 we have calculated the $\sigma$ values for both the samples according 


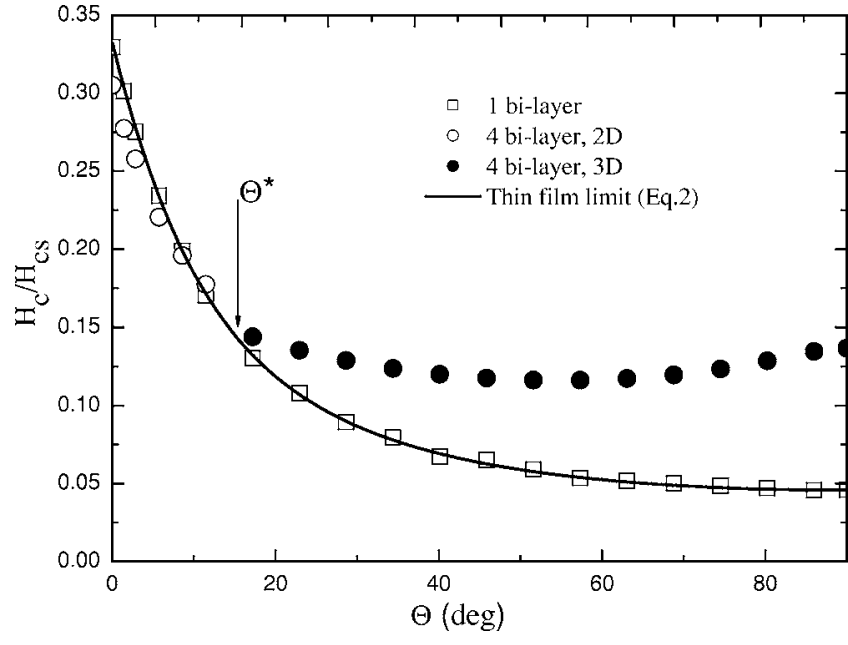

FIG. 5. Calculated $H_{\mathrm{c}}(\Theta)$ dependence for one bilayer (open squares) and four bilayer (circles) structures at $T \leqslant T^{*}$ (see text). Open (solid) circles correspond to 2D (3D) behavior. The solid line is built according to Tinkham's expression for a thin film. $H_{\mathrm{cs}}$ is the critical magnetic field of the bulk material.

to Eq. (8) and we have obtained $2 \sigma \approx 16 \mathrm{~nm}$ for sample SP (at $T=1.91 \mathrm{~K}$ ) and $2 \sigma \approx 14 \mathrm{~nm}$ (at $T=2.05 \mathrm{~K}$ ) for sample $\mathrm{NP}$, close to the $\mathrm{Nb}$ thickness $(20 \mathrm{~nm})$. Note that the values of $2 \sigma$ are also in very reasonable agreement with the $\xi(T)$ values extracted from the $H_{\mathrm{c}}(T, \Theta=\pi / 2)$ dependences. [For NP $\xi(T=2.05 \mathrm{~K}) \approx 18 \mathrm{~nm}$ and for $\mathrm{SP} \xi(T=1.91 \mathrm{~K})$ $\approx 19 \mathrm{~nm}]$. So the calculated results reproduce the main features of the experimental characteristics.

The positions of the nuclei of the superconducting phase are in close relation to the character of $H_{\mathrm{c}}(\Theta)$ dependences, as follows from the analysis of the data for our even and odd $\mathrm{Nb} / \mathrm{Pd}$ multilayers. For finite $\mathrm{S} / \mathrm{N}$ multilayers the position of the nucleus, $\langle z\rangle_{\Theta=0}=z_{0}$, accesses one of the discrete available values corresponding to the upper critical magnetic field. At low temperatures $\left(T \ll T_{\mathrm{c}}\right)$ the values of the magnetic field which correspond to different positions of nuclei are almost equal; i.e., nuclei of the superconducting phase are confined with almost the same probability in one of the superconducting layers both for nine- and ten-bilayer structures. ${ }^{17}$ And as our estimations show, the wave function decays relatively quickly within the single $\mathrm{S}$ layer. So the multilayer structure is actually reduced to one slab. It is clear that in this temperature range for both odd and even $\mathrm{S} / \mathrm{N}$ samples the same $H_{\mathrm{c}}(\Theta)$ curves should be observed. In Fig. 6 we show the $H_{\mathrm{c}}(\Theta)$ dependences for both samples measured at $T$ $=1.91 \mathrm{~K}$ (sample SP) and at $T=2.05 \mathrm{~K}$ (sample NP). It is seen that both curves are matched well.

Within the proposed physical picture it is also possible to explain the smaller $H_{\mathrm{c}}(\pi / 2)$ values obtained from the fitting procedure using Eq. (2) at $\Theta<\Theta^{*}$. Indeed, in this angular range, our sample is reduced to a simple one-bilayer $\mathrm{S} / \mathrm{N}$ structure with smaller $T_{\mathrm{c}}$ value with respect to the $3 \mathrm{D}$ multilayer structure (which has larger total $S$ layer thickness). ${ }^{42}$ The reduction of $\Theta^{*}$ with temperature (Fig. 4)

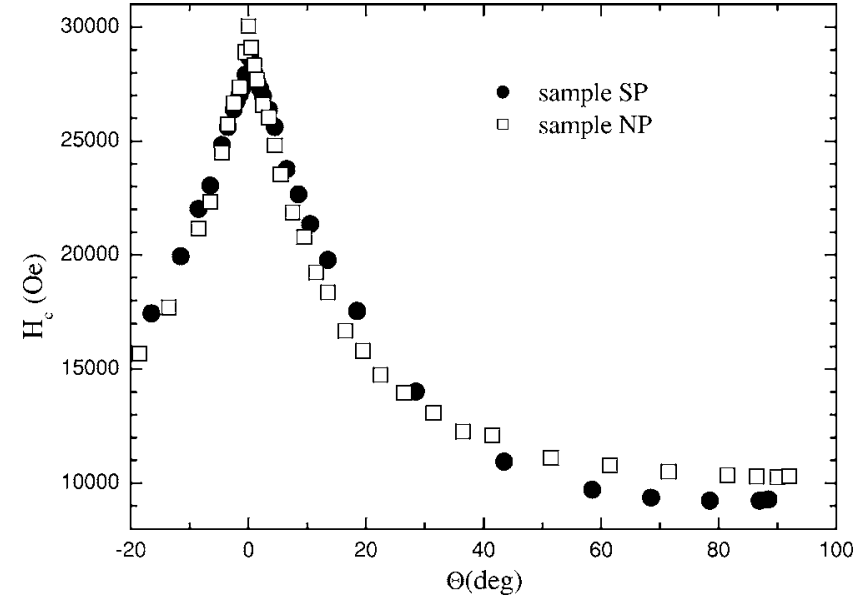

FIG. 6. $H_{\mathrm{c}}(\Theta)$ for sample SP measured at $T=1.91 \mathrm{~K}$ and for sample NP measured at $T=2.05 \mathrm{~K}$.

can also be explained within the proposed description. In fact, while increasing the temperature $\xi(T)$ increases and this reduces the localization degree of the superconducting nucleus in a parallel field, causing its delocalization at lower $\Theta^{*}$ values. This delocalization at smaller $\Theta^{*}$, finally, causes a larger change of the derivative. Moreover, the change of the derivative $\mathrm{d} H_{\mathrm{c}}(\Theta) / \mathrm{d} \Theta$ is sharper at $\Theta^{*}$ for sample NP and this is explained by the sudden change of the nucleation position of the superconducting phase which in sample SP does not actually occur.

\section{CONCLUSION}

In conclusion, we have conducted a systematic study of the angular dependences of the upper critical magnetic field for $\mathrm{Nb} / \mathrm{Pd}$ multilayers with different geometrical symmetries. Samples with an odd and even number of N/S bilayers (plus capping $\mathrm{N}$ layer) showed the change of dimensionality with the angle of application of the external magnetic field. In the range $0<\Theta<\Theta^{*}$ samples are reduced to thin-film slabs (i.e., they are two dimensional) while for angles $\Theta^{*}$ $<\Theta<\pi / 2$ samples must be considered as actual multilayers (three dimensional). The crossover angle $\Theta^{*}$ is temperature dependent due to the temperature dependence of the superconducting coherence length.

The position of the superconducting nucleus influences the $H_{\mathrm{c}}(\Theta)$ shape, revealing a stronger bend for a multilayer with an odd number of bilayers. This reflects the change of the nucleus position between $\Theta=0$ and $\Theta=\pi / 2$. At the same time for a multilayer with an even number of bilayers the nucleus position is localized at the geometrical center of the sample at both $\Theta=0$ and $\Theta=\pi / 2$, and this is reflected in a larger bidimensionality of the sample. Based on such a physical picture and using the GL approach, an expression for $H_{\mathrm{c}}(\Theta)$ was derived which seems to reasonably explain the presence of angle dimensional crossover, the measured characteristics at small $\Theta$ for different temperatures, the positions of the superconducting nucleus, and the symmetry of the $\mathrm{S} / \mathrm{N}$ multilayers. 
${ }^{1}$ B. Y. Jin and J. B. Ketterson, Adv. Phys. 38, 189 (1989); G. Blatter, M. V. Feigelman, V. B. Geshkenbein, A. I. Larkin, and V. M. Vinokur, Rev. Mod. Phys. 66, 1125 (1994); J. Flouquet and A. Buzdin, Phys. World 15, 41 (2002).

${ }^{2}$ I. Banerjee, Q. S. Yang, C. M. Falco, and I. K. Schuller, Phys. Rev. B 28, 5037 (1983).

${ }^{3}$ C. S. L. Chun, G. G. Zheng, J. L. Vicent, and I. K. Schuller, Phys. Rev. B 29, 4915 (1984).

${ }^{4}$ I. Banerjee and I. K. Schuller, J. Low Temp. Phys. 54, 501 (1984).

${ }^{5}$ J. Guimpel, M. E. de la Cruz, F. de la Cruz, H. J. Fink, O. Laborde, and J. C. Villigier, J. Low Temp. Phys. 63, 151 (1986).

${ }^{6}$ K. Kanoda, H. Mazaki, T. Yamada, N. Hosoito, and T. Shinjo, Phys. Rev. B 33, 2052 (1986); K. Kanoda, H. Mazaki, N. Hosoito, and T. Shinjo, ibid. 35, 6736 (1987).

${ }^{7}$ J. P. Locquet, W. Sevenhans, Y. Bruynseraede, H. Homma, and I. K. Schuller, IEEE Trans. Magn. 23, 1393 (1987).

${ }^{8}$ P. R. Broussard and T. H. Geballe, Phys. Rev. B 35, 1664 (1987); 37, 60 (1988).

${ }^{9}$ V. I. Dediu, V. V. Kabanov, and A. S. Sidorenko, Phys. Rev. B 49, 4027 (1994).

${ }^{10}$ C. Coccorese, C. Attanasio, L. V. Mercaldo, M. Salvato, L. Maritato, J. M. Slaughter, C. M. Falco, S. L. Prischepa, and B. I. Ivlev, Phys. Rev. B 57, 7922 (1998).

${ }^{11}$ C. Cirillo, C. Attanasio, L. Maritato, L. V. Mercaldo, S. L. Prischepa, and M. Salvato, J. Low Temp. Phys. 130, 509 (2003).

${ }^{12}$ C. Attanasio, C. Coccorese, L. V. Mercaldo, M. Salvato, L. Maritato, A. N. Lykov, S. L. Prischepa, and C. M. Falco, Phys. Rev. B 57, 6056 (1998).

${ }^{13}$ S. Takahashi and M. Tachiki, Phys. Rev. B 33, 4620 (1986).

${ }^{14}$ R. T. W. Koperdraad and A. Lodder, Phys. Rev. B 54, 515 (1996).

${ }^{15}$ C. Ciuhu and A. Lodder, Phys. Rev. B 64, 224526 (2001).

${ }^{16}$ V. N. Kushnir, S. L. Prischepa, M. L. Della Rocca, M. Salvato, and C. Attanasio, Phys. Rev. B 68, 212505 (2003).

${ }^{17}$ V. N. Kushnir, S. L. Prischepa, C. Cirillo, M. L. Della Rocca, A. Angrisani Armenio, L. Maritato, M. Salvato, and C. Attanasio, Eur. Phys. J. B 41, 439 (2004).

${ }^{18}$ V. N. Kushnir, A. Y. Petrov, and S. L. Prischepa, Fiz. Nizk. Temp. 25, 1265 (1999) [Low Temp. Phys. 25, 948 (1999)].

${ }^{19}$ K. Takanaka, L. Sakai, and H. Matueda, Phys. Rev. B 66, 012514 (2002)

${ }^{20}$ D. Saint-James and P. G. de Gennes, Phys. Lett. 7, 306 (1963).
${ }^{21}$ M. Tinkham, Phys. Rev. 129, 2413 (1963); Phys. Lett. 9, 217 (1964).

${ }^{22}$ D. Saint-James, Phys. Lett. 16, 218 (1965).

${ }^{23}$ W. E. Lawrence and S. Doniach, in Proceedings of the 12th International Conference on Low Temperature Physics, Kyoto, 1970, edited by E. Kanada (Keigaki, Tokyo, 1971), p. 361.

${ }^{24} \mathrm{~K}$. Yamafuji, E. Kusayanagi, and F. Irie, Phys. Lett. 21, 11 (1966).

${ }^{25}$ S. T. Ruggiero, T. W. Barbee, Jr., and M. R. Beasley, Phys. Rev. B 26, 4894 (1982).

${ }^{26}$ B. Y. Jin, J. B. Ketterson, E. J. McNiff, S. Foner, and I. K. Schuller, J. Low Temp. Phys. 69, 39 (1987).

${ }^{27}$ L. I. Glazman, Zh. Eksp. Teor. Fiz. 93, 1373 (1987) [Sov. Phys. JETP 66, 780 (1987)].

${ }^{28}$ V. L. Tovazhnyanskii, V. G. Cherkasova, and N. Ya. Fogel', Zh. Eksp. Teor. Fiz. 93, 1384 (1987) [Sov. Phys. JETP 66, 787 (1987)].

${ }^{29}$ M. J. Naughton, R. C. Yu, P. K. Davies, J. E. Fischer, R. V. Chamberlin, Z. Z. Wang, T. W. Jing, N. P. Ong, and P. M. Chaikin, Phys. Rev. B 38, R9280 (1988).

${ }^{30}$ R. Fastampa, M. Giura, R. Marcon, and E. Silva, Phys. Rev. Lett. 67, 1795 (1991).

${ }^{31}$ E. V. Minenko and I. O. Kulik, Fiz. Nizk. Temp. 5, 1237 (1979) [Sov. J. Low Temp. Phys. 5, 583 (1979)].

${ }^{32}$ V. M. Gvozdikov, Fiz. Nizk. Temp. 16, 5 (1990) [Sov. J. Low Temp. Phys. 16, 1 (1990)].

${ }^{33}$ K. Takanaka, J. Phys. Soc. Jpn. 58, 668 (1989); ibid. 61, 2865 (1992).

${ }^{34}$ T. Schneider and A. Schmidt, Phys. Rev. B 47, 5915 (1993).

${ }^{35}$ N. Takezawa, T. Koyama, and M. Tachiki, Physica C 207, 231 (1993).

${ }^{36}$ V. P. Mineev, Phys. Rev. B 65, 012508 (2001).

${ }^{37}$ C. Cirillo, S. L. Prischepa, M. Salvato, and C. Attanasio, Eur. Phys. J. B 38, 59 (2004).

${ }^{38}$ A. A. Golubov and A. E. Koshelev, Phys. Rev. B 68, 104503 (2003).

${ }^{39}$ M. Tinkham, Introduction to Superconductivity, 2nd ed. (McGraw-Hill, New York, 1996).

${ }^{40}$ V. N. Kushnir (unpublished).

${ }^{41}$ A. Yu. Kitaev and L. S. Levitov, Zh. Eksp. Teor. Fiz. 95, 311 (1989) [Sov. Phys. JETP 68, 176 (1989)].

${ }^{42}$ P. G. de Gennes, Rev. Mod. Phys. 36, 225 (1964).

${ }^{43}$ K. K. Likharev, Rev. Mod. Phys. 51, 101 (1979). 